\title{
On the inclusion of data from solar cycle 19 to estimate F2 layer characteristic long term trends
}

\author{
Blas F. de Haro Barbas ${ }^{1, \star}$, Ana G. Elias ${ }^{1,2}$ \\ ${ }^{1}$ Universidad Nacional de Tucumán, Departamento de Física, Lab. Física de la Atmosfera, Tucumán, Argentina \\ ${ }^{2}$ Consejo Nacional de Investigaciones Cientificas y Técnicas, CONICET, Argentina
}

\author{
Article history \\ Received September 30, 2014; accepted June 18, 2015. \\ Subject classification: \\ Ionospheric trend, EUV proxies, Forecasts, Dynamics.
}

\begin{abstract}
The effect of including solar cycle 19 (1954-1964) in ionospheric trend estimation is assessed using experimental foF 2 values. The dominant influence on the F2 layer is solar EUV radiation. In fact, around 90\% of inter-annual variance of ionospheric parameters, such as foF2, is explained by solar EUV proxies such as the sunspot number, Rz, and solar radio flux at $10.7 \mathrm{~cm}, \mathrm{~F} 10.7$. This makes necessary to filter out solar activity effects prior to long term trends estimation, which is reduced at most to the remaining $10 \%$ variance. In general solar activity is filtered assessing the residuals of a linear regression between foF 2 and $R z$, or between foF 2 and F10.7. Solar cycle 19 is a strong cycle during which Rz and F10.7 exceeded the values beyond which the ionosphere does not respond linearly to a further increase in EUV radiation. This effect, called saturation, implies a break down of the linearity between foF 2 and EUV, and results in persistent negative residuals during this period. Since solar cycle 19 is at the beginning of the time series, trends result to be positive, or less negative, than trends without considering this period. In this case the filtering process is generating a "spurious" trend in the filtered data series which may lead to erroneous conclusions. $h m F 2$ that do not present a saturation effect is also analyzed.
\end{abstract}

\section{Introduction}

To understand and predict the variability of the F2 region of the ionosphere it is essential to understand EUV variability which explains around $90 \%$ of the variation of parameters such as the critical frequency of the F2 layer, fof2, and the height of peak electron concentration, hmF2 [Chen et al. 2012]. In the study of long-term trends it is necessary then to apply filtering processes in order to filter out solar activity effect and make trends detectable.

Ionospheric trends have become a main subject since the beginning of the 1990's when the study of upper atmosphere trends gained importance in the context of global climatic change (see Lastovicka et al. [2012], and references there in). Several studies link ionospheric trends with the middle and upper atmosphere cooling due to an increase in greenhouse gases [Roble and Dickinson 1989, Rishbeth 1990, Upadhyay and Mahajan 1998]. A doubling in $\mathrm{CO}_{2}$ concentration would produce a cooling of $30-40 \mathrm{~K}$ in the thermosphere, a $20 \%-40 \%$ decrease in air density between $200-300 \mathrm{~km}$, a $\sim 15-20 \mathrm{~km}$ lowering of $\mathrm{hmF} 2$ and a worldwide decrease in foF2 less than $0.5 \mathrm{MHz}$ [Roble and Dickinson 1989, Rishbeth 1990, Rishbeth and Roble 1992]. However, the global pattern of experimental hmF2 and foF2 of several worldwide stations is highly complex and cannot be entirely reconciled with the greenhouse hypothesis. Some reasons for this are, on one side the existence of other sources of upper atmosphere trends (such as geomagnetic activity long-term variation and Earth's magnetic field secular variations) which act jointly with the greenhouse effect. On the other side, the methods used to extract trend values differ from author to author, and usually rely on some filtering process that may bias the trend results.

Filtering is a standard mathematical operation which in the case of ionosphere is used to enhance features, as it is a long-term trend, otherwise not visibly apparent in the data. However, filtering can have unexpected consequences such as the introduction of spurious oscillations or a spurious trend [Chandler and Scott 2011]. A special care is therefore required when making inferences from filtered time series.

Since there are not long-term continuous measurements of solar EUV, different indices are used to describe the variations of solar EUV irradiance and to filter ionospheric parameters. Among these indices, F10.7 and $\mathrm{Rz}$ are the most widely used.

Saturation takes place when $\mathrm{Rz}$ or F10.7 exceeds certain value, beyond which the ionosphere seems not respond to a further increase in EUV radiation [Balan et 
al. 1994, Liu et al. 2003, Ma et al. 2009]. It is linked to a break down of the linearity between foF 2 and EUV (or solar index), as reported in Liu et al. [2006, 2011]. Saturation is dominant in the equatorial ionization anomaly regions during all seasons, while at mid-latitudes it is weaker in local winter but it is still present in other seasons [Chen and Liu 2010]. Figure 1 shows foF2 measured at Slough in term of F10.7 for different months, representing different seasons. foF $2^{2}$, which is proportional to NmF2, is also shown.

The saturation effect can be clearly noticed for this mid-latitude station during local summer. During winter foF2 nearly increases linearly with F10.7 in agreement with Chen and Liu [2010] results for mid-latitudes.

Filtering methods are based on linearity, so the saturation effects may strongly influence the long-term trend reached.

This problem can be overcome in some cases using foF $2^{2}(\propto N m F 2)$ instead of foF2. But since this is not always the case another possibility is to exclude the period from the analysis.

This situation results in persistent negative residuals which will affect the trend value according to where this period of persistence is located within the whole period of analysis. If it is in the middle, it may not cause any
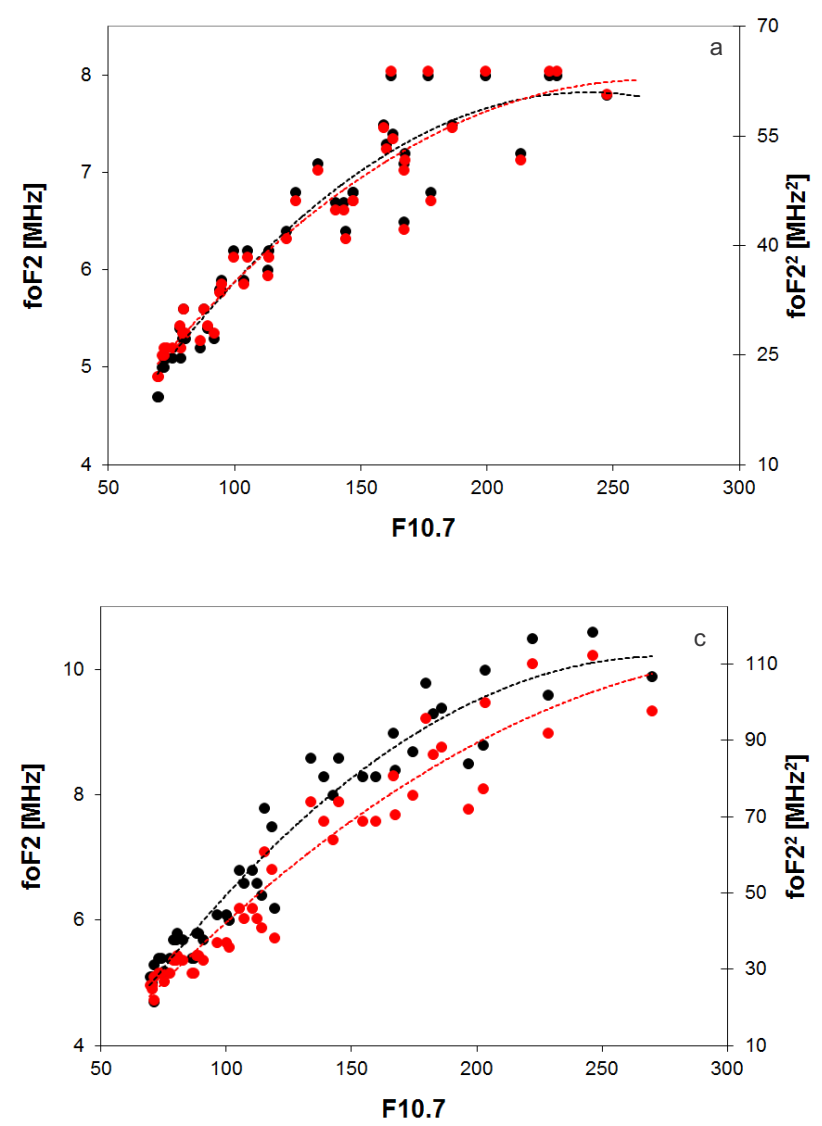

spurious results, but if is close to an extreme it will depend if it is at the beginning, inducing a spurious positive trend, or at the end inducing a spurious negative trend.

In particular, solar cycle 19 (1954-1964) has a very high maximum compared to other cycles, which presents $\mathrm{Rz}>160$ and F10.7 $>200$. Since it is at the beginning of most ionosphere time series so it should be expected a positive, or a less negative trend, than the trend without considering this period.

In the case of $\mathrm{hmF} 2$, the results obtained by Chen and Liu [2010] indicate that a linear fit can well present its variation with F10.7 in the whole range of solar activity level. This means that, contrary to what happens with foF2, trend values should not change with the period considered.

Four stations were studied: Rome, Slough, Townsville and Kokobunji. The purpose of the present study is to see the impact of solar cycle 19 in estimating trends.

\section{Data analysis}

foF2 and M(3000)F2 monthly median data for the month of June at 12 LT from Slough, Rome, Ottawa and Townsville were obtained from Damboldt and Suessmann database [2012] (ftp:/ / ftp.ips.gov.au/wdc-data/ iondata/medians/Damboldt/), for the period 1954-1995.
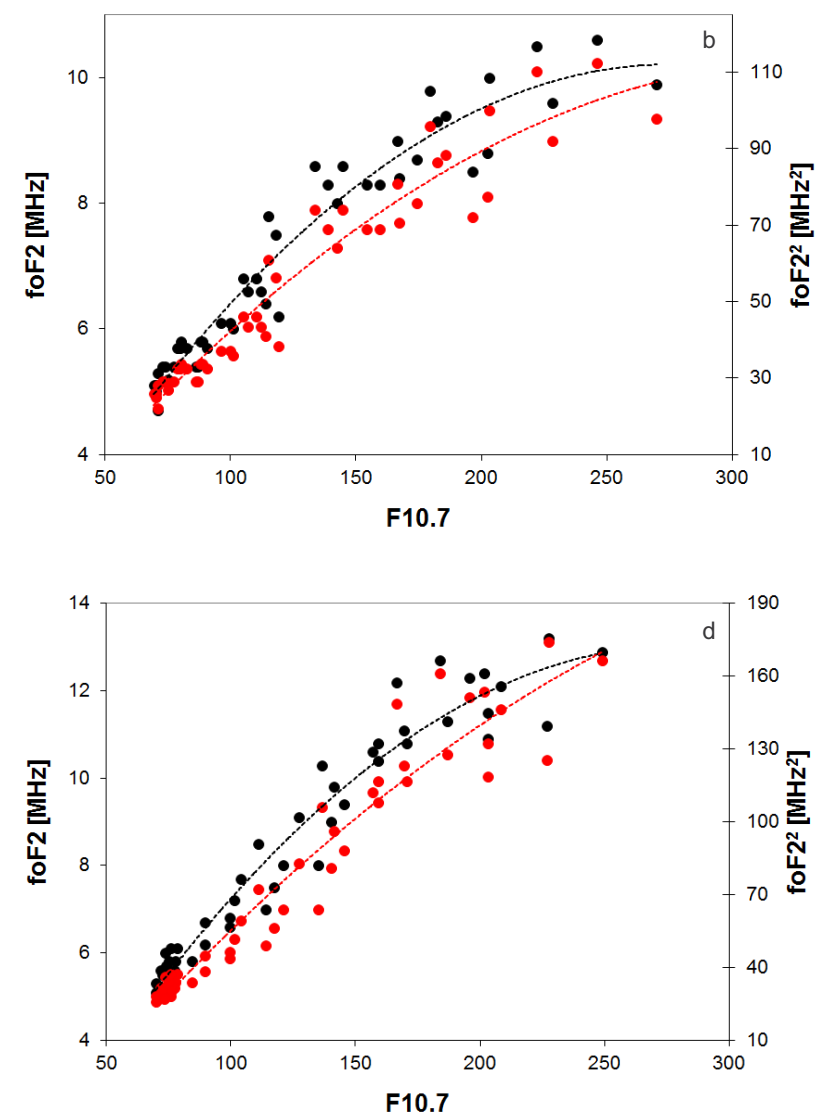

Figure 1. foF2 (black circles) measured at Slough, and foF2² (red circles) in terms of F10.7 for (a) June, (b) September, (c) December, and (d) March. Dotted lines correspond to a polynomial fit of order 2. The foF2 data are monthly median values for period 1954-1995. 
The first step in order to assess foF 2 trends is to filter out solar activity. This is done estimating the foF 2 residuals from the regression between the experimental values and the solar activity index F10.7 that is:

$$
\text { foF2res }=\text { foF2exp }-(a \text { F10.7 }+b)
$$

where $a$ and $b$ are constants determined with least squares.

Mielich and Bremer [2013] analyzed more than 100 stations in order to detect the best index to filter solar activity from foF 2 and $\mathrm{hmF} 2$, and conclude that F10.7 is a better index than $\mathrm{Rz}$.

Then a regression of foF2res with time is adjusted with least squares so that

$$
\text { foF } 2 \text { res }=\alpha t+\beta
$$

where $\alpha$ and $\beta$ are the regression coefficients, and $\alpha$ (in $\mathrm{MHz} /$ year) is the trend we are interested in.

Trend values were calculated for three different periods: (1) complete period available, 1954-1995, which includes solar cycle 19, (2) period 1954-1995 excluding periods where F10.7 exceeded 200, (3) period 1964-1995 that excludes solar cycle 19 .

Since the peak electron density, $\mathrm{NmF} 2$, in the ionosphere is linked to foF2 through:

$$
\mathrm{NmF} 2=1.24 \times 10^{4}(\mathrm{foF} 2)^{2}
$$

the same filtering process was also applied to foF $2^{2}$. foF $2^{2}$ res was estimated as

$$
\text { foF } 2^{2} \text { res }=f o F 2^{2} \exp -\left(a^{\prime} \mathrm{F} 10.7+b^{\prime}\right)
$$

followed by

$$
\text { foF } 2^{2} \text { res }=\alpha^{\prime} t+\beta^{\prime}
$$

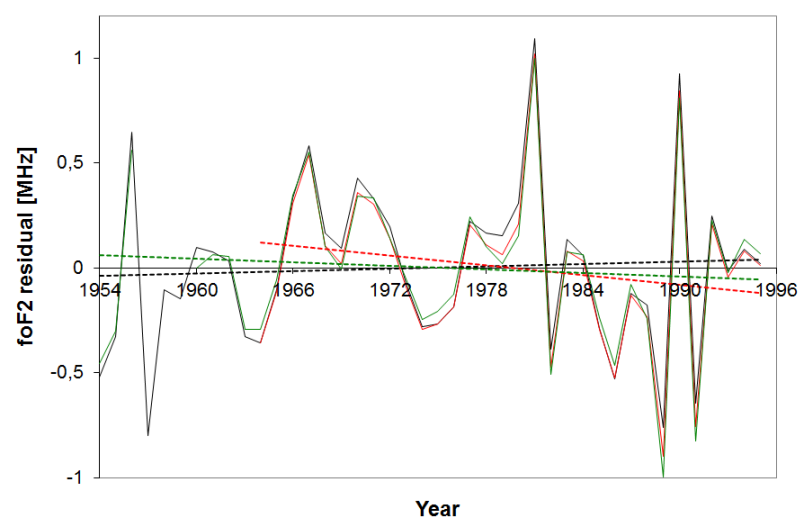

Figure 2. foF2 residual (June, 12 LT) for Slough assessed for the periods: 1954-1995 (black line), 1964-1995 (red line), 1954-1995 excluding periods where F10.7 exceeded 200 (green line). Dotted lines are the corresponding linear trends.

where $a^{\prime}, b^{\prime}, \alpha^{\prime}$ and $\beta^{\prime}$ are constants determined with least squares, and $\alpha^{\prime}$ (in $\mathrm{MHz}^{2}$ /year) is foF $2^{2}$ trend.

Figure 2 shows as an example foF 2 residuals in the case of Slough together with the linear trends for the three cases considered. Trend values, $\alpha$ and $\alpha^{\prime}$, for all the station analyzed are listed in Table 1.

Only in the case of Townsville the trend becomes positive when solar cycle 19 is not included. This is due to a relatively big positive residual in 1960 , and in a time series of 39 data it makes a difference when it is $\sim 7$ times the average absolute value of the residuals. In this case foF $2^{2}$ res $=40 \mathrm{MHz}^{2}$ while the average of $\mid f o F 2^{2}$ res $\mid$ is $6 \mathrm{MHz}^{2}$. This difference is less evident when foF2 is not squared. When only periods where F10.7 exceeded 200 are excluded, the 1960 value remains and the trend results negative.

In the case of hmF2, it was first estimated using the formula of Shimazaki [1955]:

\begin{tabular}{|c|c|c|c|c|c|c|c|c|}
\hline \multirow[b]{2}{*}{ Station } & \multicolumn{2}{|c|}{ Geographic } & \multicolumn{3}{|c|}{$\begin{array}{c}\text { foF2 trend } \\
\alpha[\mathrm{MHz} / \text { year }]\end{array}$} & \multicolumn{3}{|c|}{$\begin{array}{c}\text { foF2 } 2^{2} \text { trend } \\
\alpha\left[\mathrm{MHz}^{2} / \text { year }\right]\end{array}$} \\
\hline & Lat. & Long. & 1954-1995 & $\begin{array}{l}\text { Without } \\
\text { F10.7>200 }\end{array}$ & 1964-1995 & 1954-1995 & $\begin{array}{l}\text { Without } \\
\text { F10.7>200 }\end{array}$ & 1964-1995 \\
\hline Ottawa & 45.4 & 284.1 & 0.002 & 0.000 & -0.006 & 0.023 & -0.006 & -0.070 \\
\hline Rome & 41.8 & 12.5 & -0.002 & -0.012 & -0.022 & -0.010 & -0.134 & -0.272 \\
\hline Townsville & -19.3 & 146.7 & 0.004 & -0.001 & 0.003 & 0.011 & -0.014 & 0.094 \\
\hline Slough & 51.5 & 359.4 & 0.002 & -0.003 & -0.008 & 0.012 & -0.034 & -0.088 \\
\hline
\end{tabular}

$$
\mathrm{hmF} 2=\frac{1490}{\mathrm{M}(3000) \mathrm{F} 2}-176
$$

Trends were assessed for Slough, Rome and Townsville in the same way as in the case of foF2. Ot-

Table 1. Trend values: foF 2 and foF2 22 residual trends for four ionospheric stations calculated for three periods: 1954-1995 that includes solar cycle 19, 1954-1995 excluding periods where F10.7 exceeded 200 and 1964-1995 that do not includes cycle 19. Residuals assessed using F10.7 as solar EUV proxy. 


\begin{tabular}{|c|c|c|c|c|c|}
\hline \multirow[b]{2}{*}{ Station } & \multicolumn{2}{|c|}{ Geographic } & \multicolumn{3}{|c|}{$\begin{array}{l}\mathrm{hmF} 2 \text { trend } \\
\alpha[\mathrm{km} / \text { year }]\end{array}$} \\
\hline & Lat. & Long. & $1954-1995$ & $\begin{array}{l}\text { Without } \\
\text { F10.7>200 }\end{array}$ & 1964-1995 \\
\hline Rome & 41.8 & 12.5 & -0.28 & -0.24 & -0.27 \\
\hline Townsville & -19.3 & 146.7 & -0.23 & -0.22 & -0.19 \\
\hline Slough & 51.5 & 359.4 & -0.21 & -0.03 & 0.04 \\
\hline
\end{tabular}

Table 2. Trend values: hmF2 residual trends for three ionospheric stations calculated for three periods: 1954-1995 that includes solar cycle 19, 1954-1995 excluding periods where F10.7 exceeded 200 and 1964-1995 that do not includes cycle 19. Residuals assessed using F10.7 as solar EUV proxy.

tawa was excluded in this analysis due to data gaps. $\mathrm{hmF} 2$ trend values are listed in Table 2. Except for the case of Slough, differences in $\mathrm{hmF} 2$ trends are relatively small as expected.

\section{Discussion and conclusions}

Filtering is a crucial step in ionosphere long-term trend estimation which can lead to "spurious" trends when not properly applied to the time series under analysis.

It can be noticed from Table 1, that in all the cases, except foF $2^{2}$ for Townsville, trends become negative, or less positive, when solar cycle 19 (1954-1964) is excluded from trend estimation.
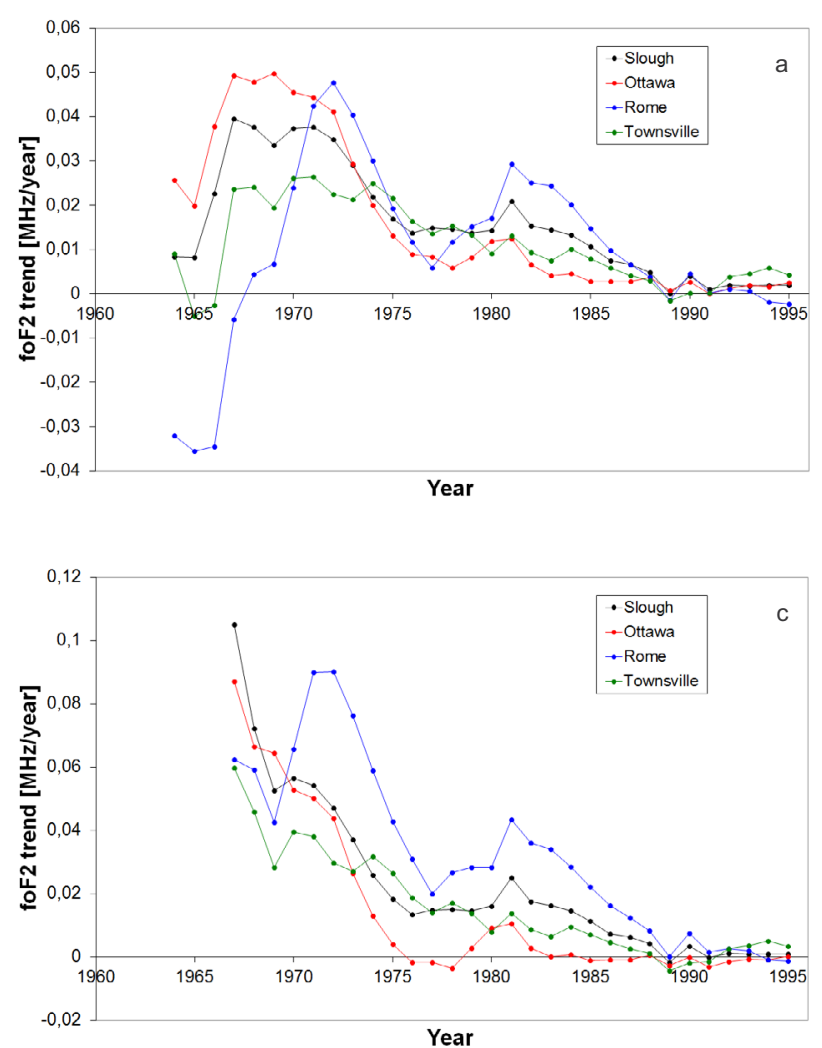

foF2 trend values for Ottawa, Townsville and Slough are in close agreement with the values obtained by Upadhyay and Mahajan [1998] who analyzed the period 1957-1990. These positive trends, together with other stations, made them conclude: "Trends were negative for some stations and positive for other stations. We conclude that ionospheric data do not provide any definitive evidence of a global long term trend".

Clilverd et al. [2003] analyzed the impact of residual periodicities on the trend values considering that filtering from a time series a periodical signal result in a damped periodical signal. They did this for $\mathrm{hmF} 2$, considering the first 10 years of the residual time series and then adding the following year and computing a new
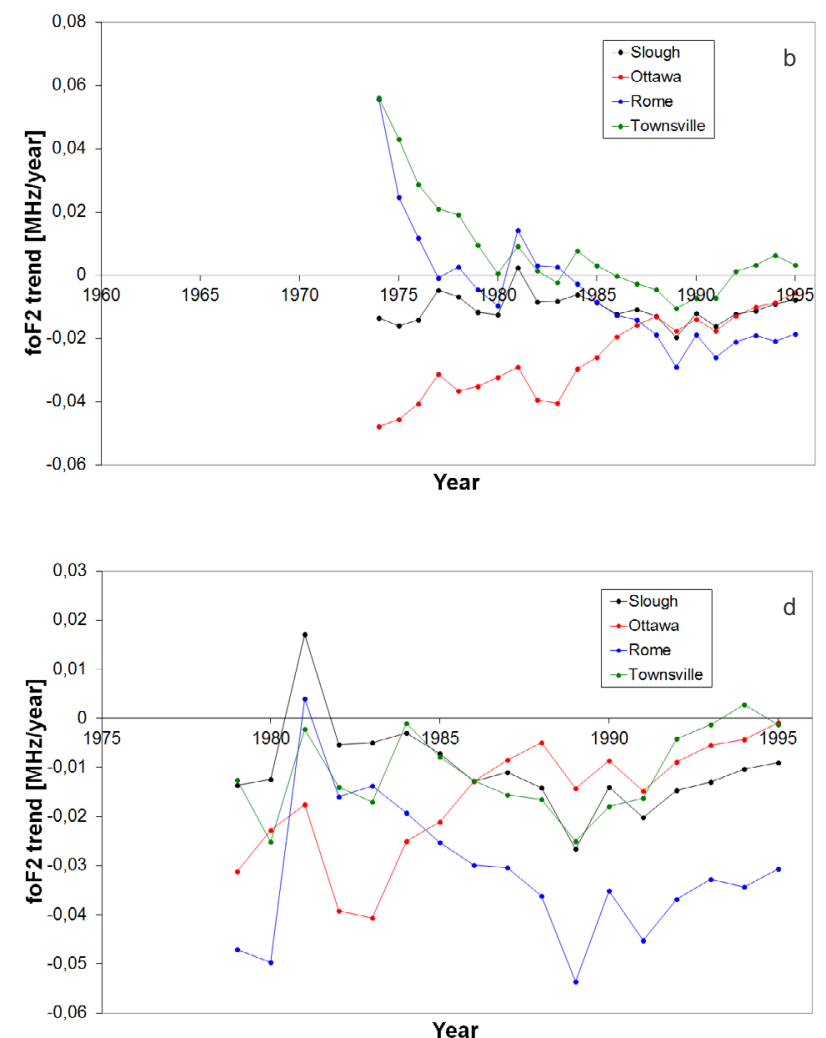

Figure 3. foF2 trend estimated for foF2 residual time series starting during (a) 1954 solar minimum, (b) 1964 solar minimum, (c) 1957 solar maximum, and (d) 1969 solar maxim considering the first 10 years and then adding the following year for a new data and adding one year at a time. The year corresponding to each trend value is the final year considered for trend estimation. 
trend, obtaining a trend values in terms of the length of the series considered. When the process began at a solar maximum the first value was negative and when it began in a solar minimum it was positive. Both trend series should result in a ringing which is gradually damped out, tending both curves to the same final value.

We tested this effect in our case for foF 2 and $\mathrm{hmF} 2$, following their procedure [Clilverd et al. 2003], that is considering the first 10 years of the residual time series and then adding the following years one at a time and computing a new trend successively. Figure 3 shows foF2 trends beginning in two minimum epochs: 1954 (cycle 19) and 1964 (cycle 20), and also in two maximum epochs: 1957 (cycle 19) and 1969 (cycle 20). As expected from our results, it makes a clear difference including or not solar cycle 19 , especially when the foF 2 residual time series begin at a maximum. We obtain similar results to those of Clilverd et al. [2003] when solar cycle 19 is not included.

When beginning at solar minimum, our results are not so clear but the ringing effect can still be noticed.

In the case of hmF2, depicted in Figure 4, we do not obtain very clear results. When $\mathrm{hmF} 2$ residuals begin at the 1954 minimum, trends begin with a positive value, in coincidence with Clilverd et al. [2003] results. However when we shift the beginning to 1964, trends begin with a negative value. In the case of solar maximum, our results do not agree with Clilverd et al. [2003]. A possible cause of this disagreement may be due to the fact that we analyzed just one month, and probably seasonality has some effect which is smoothed when annual data is considered. The difference found between stations deserves further analysis.

In conclusion, in the case of solar cycle 19, including it in the period of analysis and filtering using a linear fit between the ionosphere parameter and the solar EUV proxy, may result in less negative or even positive trends. This may erroneously lead to a disagreement with the expected effects of the increasing concentration of greenhouse gases, in favor of other mechanisms.

When trends are assessed with experimental foF2 data including solar cycle 19 care must be taken then with the filtering method used.

In conclusion, at least in the case of foF 2 the underlying "true" trend value can be affected by the filtering process due to solar EUV emissions during solar cycle 19 are not well represented by usual proxies such as F10.7.

\section{References}

Balan, N., G.J. Bailey, B. Jenkins, P.B. Rao and R.J. Moffet (1994). Variations of ionospheric ionization and related solar fluxes during an intense solar cycle, J. Geophys. Res., 99, 2243-2253.
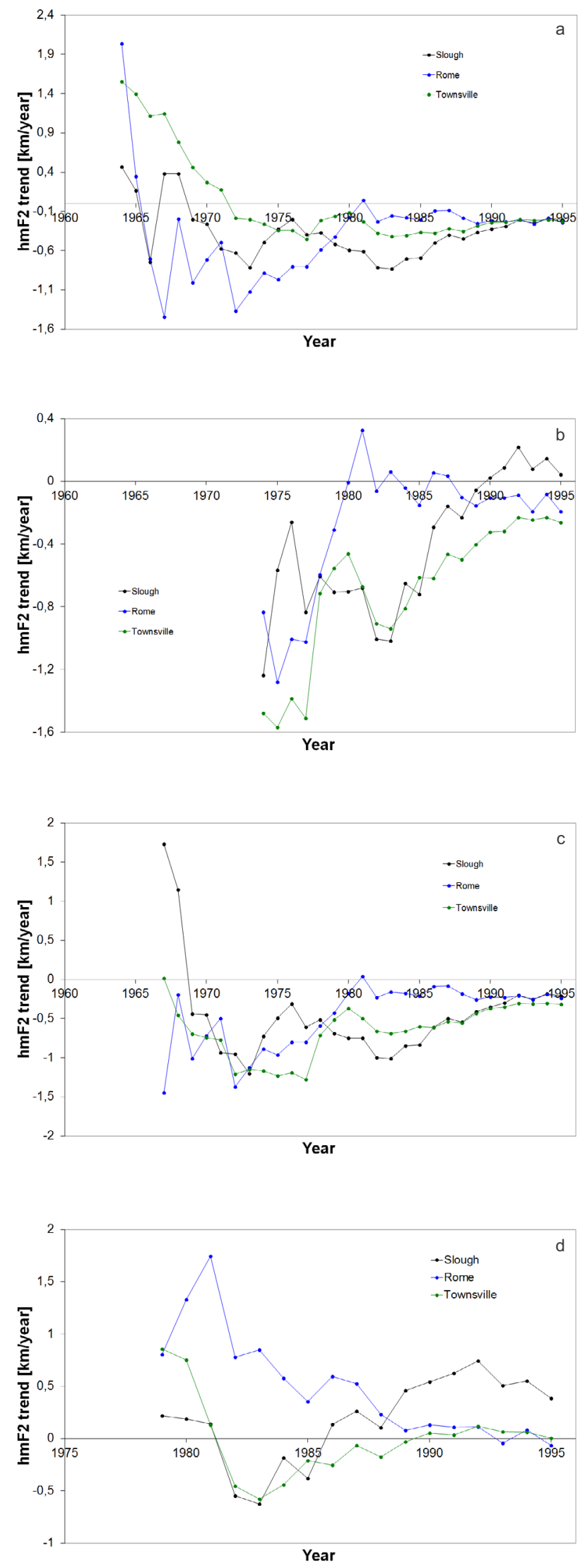

Figure 4. hmF2 trend estimated for hmF2 residual time series starting during (a) 1954 solar minimum, (b) 1964 solar minimum, (c) 1957 solar maximum, (d) and 1969 solar maximum considering the first 10 years and then adding the following year for a new data and adding one year at a time. The year corresponding to each trend value is the final year considered for trend estimation. 
Chandler, R.E., and E.M. Scott (2011). Statistical Methods for Trend Detection and Analysis in the Environmental Sciences, John Wiley \& Sons, London.

Chen, Y., and L. Liu (2010). Further study on the solar activity variation of daytime NmF2, J. Geophys. Res., 115, A12337; doi:10.1029/2010JA015847.

Chen, Y., L. Liu and W. Wan (2012). The discrepancy in solar EUV-proxy correlations on solar cycle and solar rotation timescales and its manifestation in the ionosphere, J. Geophys. Res., 117; doi:10.1029/2011J A017224.

Clilverd, M.A., T. Ulich and M.J. Jarvis (2003). Residual solar cycle influence on trends in ionospheric F2layer peak height, J. Geophys. Res., 108 (A12), 1450; doi:10.1029/2003JA009838.

Damboldt, T., and P. Suessmann (2012). Consolidated Database of Worldwide Measured Monthly Medians of Ionospheric Characteristics foF2 and $\mathrm{M}(3000) \mathrm{F} 2$, INAG Bulletin on the Web, INAG-73; http: / / www.ips.gov.au/IPSHosted/INAG/ web73/2012/damboldt_consolidated_database.pdf.

Lastovicka, J., S.C. Solomon and L. Qian (2012). Trends in the Neutral and Ionized Upper Atmosphere, Space Sci. Rev., 168, 113-145.

Liu, J.Y., Y.I. Chen and J.S. Lin (2003). Statistical investigation of the saturation effect in the ionospheric foF2 versus sunspot, solar radio noise, and solar EUV radiation, J. Geophys. Res., 108 (A2), 1067; doi:10.1029/2001JA007543.

Liu, L., W. Wan, B. Ning, O.M. Pirog and V.I. Kurkin (2006). Solar activity variations of the ionospheric peak electron density, J. Geophys. Res., 111, A08304; doi:10.1029/2006JA011598.

Liu, L., W. Wan, Y. Chen and H. Le (2011). Solar activity effects of the ionosphere: A brief review, Chinese Sci. Bull., 56, 1202-1211; doi:10.1007/s11434-0104226-9.

Ma, R., J. Xu, W. Wang and W. Yuan (2009). Seasonal and latitudinal differences of the saturation effect between ionospheric $\mathrm{NmF} 2$ and solar activity indices, J. Geophys. Res., 114, A10303; doi:10.1029/2009JA 014353.

Mielich, J., and J. Bremer (2013). Long-term trends in the ionospheric $\mathrm{F} 2$ region with different solar activity indices, Annales Geophysicae, 31, 291-303.

Rishbeth, H. (1990). A greenhouse effect in the ionosphere?, Planetary and Space Sciences, 38, 945-948.

Rishbeth, H., and R.G. Roble (1992). Cooling of the upper atmosphere by enhanced greenhouse gases. Modeling of the thermospheric and ionospheric effects, Planetary and Space Sciences, 40, 1011-1026.

Roble, R.G., and R.E. Dickinson (1989). How will changes in carbon dioxide and methane modify the mean structure of the mesosphere and thermosphere?, Geophys. Res. Lett., 16, 1441-1444.

Shimazaki, T. (1955). World wide daily variations in the height of the maximum electron density in the ionospheric F2 layer, J. Radio Res. Labs., Japan, 2, 85-97.

Upadhyay, H.O., and K.K. Mahajan (1998). Atmospheric greenhouse effect and ionospheric trends, Geophys. Res. Lett., 25, 3375-3378.
Corresponding author: Blas F. de Haro, Universidad Nacional de Tucumán, Departamento de Física, Tucumán, Argentina; email: blasdeharo2000@yahoo.com.ar.

(C) 2015 by the Istituto Nazionale di Geofisica e Vulcanologia. All rights reserved. 